\title{
Testing a simplified version of Einstein's equations for numerical relativity
}

\author{
Gregory B. Cook \\ Center for Radiophysics and Space Research, Cornell University, Ithaca, New York 14853 \\ Stuart L. Shapiro \\ Center for Astrophysics and Relativity, 326 Siena Drive, Ithaca, New York 14850 \\ Saul A. Teukolsky* \\ Center for Radiophysics and Space Research, Cornell University, Ithaca, New York 14853
}

(Received 5 December 1995)

\begin{abstract}
Solving dynamical problems in general relativity requires the full machinery of numerical relativity. Wilson has proposed a simpler but approximate scheme for systems near equilibrium, such as binary neutron stars. We test the scheme on isolated, rapidly rotating, relativistic stars. Since these objects are in equilibrium, it is crucial that the approximation works well if we are to believe its predictions for more complicated systems such as binaries. Our results are very encouraging. [S0556-2821(96)00710-2]

PACS number(s): 04.25.Dm, 04.40.Dg
\end{abstract}

\section{INTRODUCTION}

Some of the most interesting unsolved problems in general relativity require full dynamical solutions of Einstein's equations in three spatial dimensions. Such solutions have to be found numerically, and this is only barely becoming technically feasible. An important set of problems in this category is the binary coalescence of black holes and the binary coalescence of neutron stars. Such events are expected to be a significant source of gravitational waves that will be detectable by new generations of detectors such as the Laser Interferometric Gravitational Wave Observatory (LIGO).

In Newtonian physics, binary stars can orbit in an equilibrium system. In general relativity, by contrast, a binary system loses energy by gravitational wave emission. The orbit shrinks, and the two stars ultimately coalesce. Though this is clearly not an equilibrium situation, the orbital decay occurs on a much longer time scale than an orbital period, at least up until the last plunging orbit when the stars are very close. Preliminary calculations of binary coalescence and gravitational collapse suggest that the amount of energy radiated gravitationally is small. Thus, even when the system becomes highly dynamical and far from equilibrium, one might expect that it is still the nonradiative part of the gravitational field that controls the evolution.

Wilson [1-3] has proposed an approximation scheme that tracks the evolution of coalescing binary neutron stars without solving the full dynamical Einstein field equations. The method may also be applicable to binary black hole systems [4]. The scheme applies to systems that are either in or near equilibrium, in which case a reduced set of Einstein's equation should adequately describe the system. For example, a binary system is near equilibrium as long as the emission of gravitational radiation is small. In strict equilibrium, as in the case of a single rotating star, there is a coordinate frame in

\footnotetext{
*Also at Departments of Physics and Astronomy, Cornell University.
}

which the first and second time derivatives of the metric are zero. In the $3+1$ formalism, this means in particular that the time derivatives of the three-metric $\gamma_{i j}$ and the extrinsic curvature $K_{i j}$ are zero. In quasiequilibrium, the time derivatives are small, and the metric and extrinsic curvature will not depart significantly from their initial values. Wilson's approximation consists in setting time derivatives exactly equal to zero in a selected subset of Einstein's equations, and ignoring the remaining dynamical equations. This approximation results in a smaller, more tractable set of field equations. Part of the strategy for selecting the subset of Einstein's equations is to guarantee that $\gamma_{i j}$ and $K_{i j}$ are solutions of the initial-value (or constraint) equations. Wilson has proposed evolving the system through a sequence of initial-value problems by solving the full dynamical equations for the matter in the instantaneous background metric, and then updating the metric quantities at each time step by re-solving the selected subset of Einstein's equations. We will outline below a simpler method to track the evolution, which exploits the near equilibrium of the matter as well.

As compelling as this idea sounds, it is impossible to calibrate the approximation without comparing it with solutions to the exact equations. No such exact solutions exist for realistic, dynamical three-dimensional cases. Only recently has it become possible to solve Einstein's equations numerically for interesting two-dimensional problems. In fact, it is only in the last few years that as simple a problem as the equilibrium structure of a rapidly rotating relativistic star could be thoroughly investigated. In this paper, we use these rotating equilibrium solutions to calibrate Wilson's approximation scheme. This is the simplest case for which the approximation scheme is different from the exact equations. Because the system is a true equilibrium, it is clearly necessary that the approximation work well in this case. Only then will we have confidence that the method is at all useful in more complicated situations such as binary systems.

\section{BASIC EQUATIONS}

A general metric may be written in $3+1$ form as

$$
d s^{2}=-\alpha^{2} d t^{2}+\gamma_{i j}\left(d x^{i}+\beta^{i} d t\right)\left(d x^{j}+\beta^{j} d t\right) .
$$


The dynamical equation for $\gamma_{i j}$ is

$$
\partial_{t} \gamma_{i j}=-2 \alpha K_{i j}+D_{i} \beta_{j}+D_{j} \beta_{i}
$$

where $D_{i}$ denotes a covariant derivative with respect to $\gamma_{i j}$. The trace of this equation is

$$
\partial_{t} \ln \gamma^{1 / 2}=-\alpha K+D_{i} \beta^{i}
$$

where $\gamma=\operatorname{det} \gamma_{i j}$ and $K=K^{i}{ }_{i}$. The trace-free part of Eq. (2) is

$$
\begin{aligned}
\gamma^{1 / 3} \partial_{t}\left(\gamma^{-1 / 3} \gamma_{i j}\right)= & -2 \alpha\left(K_{i j}-\frac{1}{3} \gamma_{i j} K\right)+D_{i} \beta_{j}+D_{j} \beta_{i} \\
& -\frac{2}{3} \gamma_{i j} D_{k} \beta^{k}
\end{aligned}
$$

We fix the six components of the extrinsic curvature $K_{i j}$ by demanding that each data slice be a maximal slice and that the left-hand side of Eq. (4) be equal to zero. This gives

$$
K=0 \text {, }
$$

and

$$
2 \alpha K_{i j}=D_{i} \beta_{j}+D_{j} \beta_{i}-\frac{2}{3} \gamma_{i j} D_{k} \beta^{k}
$$

Note that $\partial_{t} \gamma \neq 0$ unless $D_{i} \beta^{i}=0$.

To solve the Hamiltonian constraint equation, it is convenient to use a conformal decomposition of the spatial metric. To satisfy the demand that the left-hand side of Eq. (4) be zero, we choose the metric to be conformally flat [5] so that $\gamma^{-1 / 3} \gamma_{i j}=f_{i j}$, where $f_{i j}$ is the flat metric in whatever coordinate system is used. Therefore, we decompose the spatial metric as

$$
\gamma_{i j}=\Phi^{4} f_{i j}
$$

The conformal factor $\Phi$ is determined then by the Hamiltonian constraint

$$
\nabla^{2} \Phi=-\frac{1}{8} \Phi^{5} K^{i j} K_{i j}-2 \pi \Phi^{5} \rho
$$

where the source term is

$$
\rho=n^{a} n^{b} T_{a b} .
$$

Here $n^{a}$ is the normal vector to a $t=$ constant slice, $T_{a b}$ is the stress-energy tensor, and $\nabla^{2}$ is the flat-space Laplacian. Note that although indices $i, j, \ldots$ range over $1, \ldots, 3$, indices $a, b, \ldots$ range over $0, \ldots, 3$.

The shift vector is determined by substituting Eq. (6) into the momentum constraint

$$
D_{j} K^{i j}=8 \pi S^{i}
$$

where

$$
S^{a}=-\gamma_{b}^{a} n_{c} T^{b c}
$$

We use the results that for a conformally flat metric we may write

$$
\begin{aligned}
D^{j} \beta^{i}+D^{i} \beta^{j}-\frac{2}{3} \gamma^{i j} D_{k} \beta^{k} & \\
& =\Phi^{-4}\left[\nabla^{j} \beta^{i}+\nabla^{i} \beta^{j}-\frac{2}{3} f^{i j} \nabla_{k} \beta^{k}\right],
\end{aligned}
$$

and for $K=0$,

$$
D_{j} K^{i j}=\Phi^{-10} \nabla_{j}\left(\Phi^{10} K^{i j}\right)
$$

where $\nabla_{j}$ denotes the covariant derivative in flat space. Thus Eq. (10) becomes

$$
\begin{aligned}
\nabla^{2} \beta^{i}+\frac{1}{3} \nabla^{i}\left(\nabla_{j} \beta^{j}\right)= & \left(\frac{1}{\alpha} \nabla_{j} \alpha-\frac{6}{\Phi} \nabla_{j} \Phi\right) \\
& \times\left(\nabla^{j} \beta^{i}+\nabla^{i} \beta^{j}-\frac{2}{3} f^{i j} \nabla_{k} \beta^{k}\right) \\
& +16 \pi \alpha \Phi^{4} S^{i}
\end{aligned}
$$

This equation can be simplified to two equations, one involving a vector Laplacian and the other a scalar Laplacian, by setting

$$
\beta^{i}=G^{i}-\frac{1}{4} \nabla^{i} B
$$

Then the two equations that must be solved are

$$
\begin{aligned}
\nabla^{2} G^{i}= & \left(\frac{1}{\alpha} \nabla_{j} \alpha-\frac{6}{\Phi} \nabla_{j} \Phi\right)\left(\nabla^{j} \beta^{i}+\nabla^{i} \beta^{j}-\frac{2}{3} f^{i j} \nabla_{k} \beta^{k}\right) \\
& +16 \pi \alpha \Phi^{4} S^{i}
\end{aligned}
$$

and

$$
\nabla^{2} B=\nabla_{i} G^{i}
$$

Though we are not imposing the full set of dynamical equations for the evolution of $K_{i j}$, we do have the freedom to preserve the maximal slicing condition (5) by requiring $\partial_{t} K=0$. The resulting equation can also be written with a simple Laplacian by using Eq. (8). The result is the lapse equation

$$
\nabla^{2}(\alpha \Phi)=(\alpha \Phi)\left[\frac{7}{8} \Phi^{4} K_{i j} K^{i j}+2 \pi \Phi^{4}(\rho+2 S)\right],
$$

where

$$
S=\gamma^{i j} T_{i j}
$$

The above field equations, in combination with the matter equations to be discussed below, form a coupled nonlinear set that must be solved by iteration. The boundary conditions for the field quantities follow from asymptotic flatness; the specific form depends on the application. We are especially interested in uniformly rotating configurations such as binary neutron stars in synchronous orbit. For such systems we work in a corotating coordinate system so that there is no time variation of the fields (in the near equilibrium approximation of the method). Following Wilson [2], we can implement this by replacing Eq. (15) with 


$$
\beta^{i}=G^{i}-\frac{1}{4} \nabla^{i} B+(\mathbf{\Omega} \times \mathbf{r})^{i}
$$

which leaves Eqs. (17) and (16) unchanged. Here $\boldsymbol{\Omega}$ is the constant angular velocity of the system.

For the matter, we will consider a perfect fluid for which

$$
T_{a b}=\left(\rho_{0}+\rho_{i}+P\right) U_{a} U_{b}+P g_{a b} .
$$

Here $\rho_{0}$ is the rest-mass density, $\rho_{i}$ is the internal energy density, $P$ is the pressure, and $U^{a}$ is the fluid four-velocity. For this source, the density $\rho$ in Eq. (9) is

$$
\rho=\left(\rho_{0}+\rho_{i}+P\right)\left(\alpha U^{t}\right)^{2}-P,
$$

the momentum source $S^{i}$ in Eq. (11) is

$$
S^{i}=\left(\rho_{0}+\rho_{i}+P\right)\left(\alpha U^{t}\right) \gamma^{i j} U_{j},
$$

and the source term $S$ in Eq. (19) is

$$
S=\left(\rho_{0}+\rho_{i}+P\right)\left[\left(\alpha U^{t}\right)^{2}-1\right]+3 P .
$$

We treat fluids that are in uniform rotation, for which the four-velocity $U^{a}$ is given by

$$
\vec{U}=U^{t}\left(\frac{\partial}{\partial t}+\Omega \frac{\partial}{\partial \phi}\right) .
$$

The normalization condition $\vec{U} \cdot \vec{U}=-1$ gives

$$
\alpha U^{t}=\left(1+\Phi^{-4} f^{i j} U_{i} U_{j}\right)^{1 / 2}
$$

Now consider the equations for the matter in the near equilibrium approximation. The key approximation is that in the corotating frame there is a Killing vector that is timelike everywhere. In the nonrotating coordinates, this vector can be written as

$$
\vec{\xi}=\frac{\partial}{\partial t}+\Omega \frac{\partial}{\partial \phi}
$$

Because the four-velocity (25) is proportional to a Killing vector, the matter equations may be integrated to give the hydrostatic equilibrium result [6]

$$
\frac{U^{t}}{h}=\text { const, }
$$

where

$$
\ln h \equiv \int \frac{d P}{\rho_{0}+\rho_{i}+P} .
$$

For a polytropic equation of state

$$
P=K \rho_{0}^{\Gamma},
$$

where $K$ and $\Gamma$ are constants, we have

$$
\rho_{i}=\frac{P}{\Gamma-1}, \quad h=\frac{\rho_{0}+\rho_{i}+P}{\rho_{0}} .
$$

In this approximation, we have reduced all of the hydrodynamics to a single algebraic equation, Eq. (28).

\section{AXISYMMETRIC ROTATING STAR: EQUATIONS}

To calibrate the method, we apply it to a true equilibrium system in axisymmetry and compare with the complete numerical solution found with no approximations. For this purpose, we use models of rotating neutron stars supported by a polytropic equation of state. Fully relativistic models have been constructed by several authors (see Refs. [7-9] and references therein). Solving Einstein's equations for these stars is nontrivial numerically. It is only the recent availability of such solutions that makes this calibration feasible.

In spherical polar coordinates and axisymmetry, we find that Eqs. (16) and (17) are satisfied by setting the quantity $B$ of Eq. (15) to zero and with the only nonzero component of the shift vector $\beta^{\phi} \equiv \beta$. Note that this implies, not only that the left-hand side of Eq. (4) is zero, but also that $\partial_{t} \gamma=0$. This means that we are finding a stationary solution of the approximate equations. Given this solution for the shift vector, the term $K_{i j} K^{i j}$ appearing in Eqs. (8) and (18) is given by

$$
K_{i j} K^{i j}=\frac{\sin ^{2} \theta}{2 \alpha^{2}}\left(r^{2} \beta_{, r}^{2}+\beta_{, \theta}^{2}\right),
$$

where commas denote partial derivatives. Only the $\phi$ component of the vector Eq. (16) is nontrivial, and becomes the scalar equation

$$
\begin{aligned}
{\left[\nabla^{2}+\frac{2}{r} \frac{\partial}{\partial r}+\frac{2 \cot \theta}{r^{2}} \frac{\partial}{\partial \theta}\right] \beta=} & \left(\frac{1}{\alpha} \frac{\partial \alpha}{\partial r}-\frac{6}{\Phi} \frac{\partial \Phi}{\partial r}\right) \frac{\partial \beta}{\partial r} \\
& +\frac{1}{r^{2}}\left(\frac{1}{\alpha} \frac{\partial \alpha}{\partial \theta}-\frac{6}{\Phi} \frac{\partial \Phi}{\partial \theta}\right) \frac{\partial \beta}{\partial \theta} \\
& +\frac{16 \pi \alpha}{r^{2} \sin ^{2} \theta} S_{\phi} .
\end{aligned}
$$

The four-velocity components appearing in the matter sources are given by

$$
U^{t}=\left[\alpha^{2}-\Phi^{4} r^{2} \sin ^{2} \theta(\beta+\Omega)^{2}\right]^{-1 / 2},
$$

$$
U_{\phi}=\Phi^{4} r^{2} \sin ^{2} \theta U^{t}(\beta+\Omega) .
$$

The above equations turn out to be simplified versions of the exact equations for stationary, axisymmetric configurations given by Cook, Shapiro, and Teukolsky [7] (CST [10]). The exact metric has four nonzero metric coefficients, denoted by $\gamma, \rho, \alpha$, and $\omega$ by CST, though the approximate metric here has only three: $\alpha, \beta$, and $\Phi$. Thus even though there is no dynamics in the field, and even though the equation of hydrostatic equilibrium for the matter is rigorously 
obeyed, the Wilson scheme is still an approximation for this problem. The correspondence between the approximate and exact metric coefficients is given by

$$
\begin{gathered}
\alpha^{2}=e^{\gamma+\rho}, \\
\Phi^{4}=e^{\gamma-\rho}=e^{2 \alpha_{\mathrm{CST}}}, \\
\beta=-\omega .
\end{gathered}
$$

The fluid velocity $v$ in the zero angular momentum observer (ZAMO) frame used by CST is given by

$$
\left(\alpha U^{t}\right)^{2}=\frac{1}{1-v^{2}}
$$

In spherical symmetry, the approximate scheme reduces to the exact scheme, with two nonzero metric coefficients. We will now quantify the degree of error in the nonspherical axisymmetric case.

We can take over the numerical scheme of CST to solve the approximate equilibrium equations. In fact, the structure of the equations is very close in that they involve the same differential operators on the left-hand sides. In particular, Eqs. (8) and (18) involve $\nabla^{2}$, as in Eq. (3) of CST, and the operator in Eq. (33) is the same as that in Eq. (5) of CST. Therefore the solution is computed as in Eqs. (27) and (29)

TABLE I. Quantities characterizing a "normal" evolutionary sequence of $n=0.5$ polytropic neutron star

\begin{tabular}{|c|c|c|c|c|c|c|c|c|c|c|}
\hline $\bar{\epsilon}_{c}^{\mathrm{a}}$ & $\bar{\Omega}^{\mathrm{b}}$ & $\overline{I^{\mathrm{c}}}$ & $\bar{M}^{\mathrm{d}}$ & $\bar{M}_{0}^{\mathrm{e}}$ & $T / W^{\mathrm{f}}$ & $\bar{R}_{e}^{\mathrm{g}}$ & $e^{\mathrm{h}}$ & $\omega_{c} / \Omega_{c}{ }^{\mathrm{i}}$ & $v / c^{\mathrm{j}}$ & $Z_{p}{ }^{\mathrm{k}}$ \\
\hline 1.0000 & & 4 & & 1 & & 37 & & 7 & & 0.5726 \\
\hline - & 000 & 4 & & & & & & & & 5725 \\
\hline .9029 & 0.3339 & 0.01069 & 0.1237 & 84 & .0150 & 0.4274 & 0.271 & 0.7291 & 1594 & 0.5660 \\
\hline- & 0.3332 & 01068 & 0.1 & 34 & 19 & 0.4 & & & & 0.5658 \\
\hline 0.815 & & 44 & & & & & & & & 0.5641 \\
\hline - & 1 & 1 & & 4 & & & 23 & & & 0.5638 \\
\hline 0.7360 & 0.5923 & 0.01237 & 0.1 & 0.1484 & $0.0 c$ & 0.4 & 32 & 0 & & 0.5623 \\
\hline - & 0.5927 & 0.012 & 0.1 & & & 0.4 & & & & 620 \\
\hline 0.6645 & & 2 & 0.1 & & & & & & & 589 \\
\hline - & 0 & & & & & & & & & 587 \\
\hline 0.6000 & 0.7040 & 0.01496 & 0.1 & 0.1 & 4 & 0.5 & 07 & & & 0.5526 \\
\hline- & 0.7064 & 0.01 & 0.1 & 0.14 & 2 & 0.5 & 0.712 & & & 0.5527 \\
\hline 0.5417 & & & & & & & & & & \\
\hline- & 0.7256 & 0.01665 & 0.1288 & 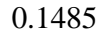 & 13 & 0.5 & 82 & & & 0.5426 \\
\hline .5148 & 0.7226 & 0.01795 & 0.1293 & 0.1484 & 0.15 & 0.6 & 0.817 & & & 0.5348 \\
\hline - & 0.7260 & 0.01777 & 0.1 & 0.1 & 0 . & 0.6 & 0.820 & & & 0.5351 \\
\hline .5115 & & & & & & & & & & \\
\hline - & 0.72 & & & & & & & & & 0.5339 \\
\hline 0.5098 & 0.7218 & 0.01 & 0.1 & 0.1 & 0. & 0.6 & 30 & 0. & 4 & 0.5332 \\
\hline- & 0.7249 & 0.01799 & 0.1 & 0.14 & 1 & 0.6 & 33 & 4 & 37 & 0.5331 \\
\hline 0.5094 & 0.7216 & 0.01822 & & & & 0.6 & 0.832 & & & 0.5330 \\
\hline - & 0.7248 & & & & & & & & & 0.5328 \\
\hline 0.5094 & 0.7216 & 0.01 & 0.1294 & 0.1 & 0. & 0.6 & 32 & 0. & 61 & 0.5330 \\
\hline- & 0.7248 & & 0.1294 & & & 0.6302 & 0.835 & & & 0.5328 \\
\hline .5094 & 0.7216 & & 0.1294 & & & 0.6333 & 0.832 & & 0.5062 & 0.5330 \\
\hline - & 0.7248 & 0.01800 & 0.1294 & 0.1484 & 0.1581 & 0.6302 & 0.835 & 0.6332 & 0.5066 & 0.5328 \\
\hline
\end{tabular}
models. For each value of the central energy density $\bar{\epsilon}$ we first display the results from solving the exact equations. Below this, indicated with a dash in the energy density column, are the results obtained by solving the approximate equations.

${ }^{a}$ Central energy density.

${ }^{\mathrm{b}}$ Angular velocity measured at infinity.

${ }^{\mathrm{c}}$ Moment of inertia.

${ }^{\mathrm{d}}$ Total mass energy.

${ }^{\mathrm{e}}$ Rest mass.

${ }^{\mathrm{f}}$ Rotational kinetic energy over gravitational binding energy.

${ }^{\mathrm{g}}$ Circumferential radius.

${ }^{\mathrm{h}}$ Eccentricity.

${ }^{\mathrm{i}}$ Measure of frame dragging.

${ }^{\mathrm{j}}$ Matter velocity at equator.

${ }^{\mathrm{k}}$ Polar redshift. 
of CST. The nondimensionalized source terms analogous to Eq. (30) of CST are

$$
\begin{aligned}
\widetilde{S}_{\Phi}(s, \mu)= & -\frac{1}{16} \frac{\Phi^{7}}{(\alpha \Phi)^{2}}\left(1-\mu^{2}\right)\left(\frac{s}{1-s}\right)^{2}\left\{\left[(1-s) s \hat{\omega}_{, s}\right]^{2}\right. \\
& \left.+\left(1-\mu^{2}\right) \hat{\omega}_{, \mu}^{2}\right\}-2 \pi \Phi^{5} \bar{r}_{e}^{2}\left(\frac{s}{1-s}\right)^{2} \\
& \times\left[\left(\overline{\rho_{0}}+\overline{\rho_{i}}+\bar{P}\right) \frac{1}{1-v^{2}}-\bar{P}\right], \\
\widetilde{S}_{\alpha \Phi}(s, \mu)= & \alpha \Phi \llbracket \frac{7}{16} \frac{\Phi^{6}}{(\alpha \Phi)^{2}}\left(1-\mu^{2}\right)\left(\frac{s}{1-s}\right)^{2} \\
& \times\left\{\left[(1-s) s \hat{\omega}_{, s}\right]^{2}+\left(1-\mu^{2}\right) \hat{\omega}_{, \mu}^{2}\right\} \\
& +2 \pi \Phi^{4} \bar{r}_{e}^{2}\left(\frac{s}{1-s}\right)^{2}\left[\left(\bar{\rho}_{0}+{\overline{\rho_{i}}}^{2} \bar{P}\right) \frac{1}{1-v^{2}}-\bar{P}\right] \\
& +4 \pi \Phi^{4} \bar{r}_{e}^{2}\left(\frac{s}{1-s}\right)^{2}\left[\left(\bar{\rho}_{0}+{\overline{\rho_{i}}}^{2} \bar{P}\right) \frac{v^{2}}{1-v^{2}}\right. \\
& +3 \bar{P}] \rrbracket,
\end{aligned}
$$

and the source term analogous to Eq. (32) of CST is

$$
\begin{aligned}
\widetilde{S}_{\hat{\omega}}(s, \mu)= & s^{2}(1-s)^{2}\left[\frac{1}{\alpha \Phi}(\alpha \Phi)_{, s}-\frac{7}{\Phi} \Phi_{, s}\right] \hat{\omega}_{, s}+\left(1-\mu^{2}\right) \\
& \times\left[\frac{1}{\alpha \Phi}(\alpha \Phi)_{, \mu}-\frac{7}{\Phi} \Phi, \mu\right] \hat{\omega}_{, \mu} \\
& -16 \pi \Phi^{4} \bar{r}_{e}^{2}\left(\frac{s}{1-s}\right)^{2} \frac{\bar{\rho}_{0}+\bar{\rho}_{i}+\bar{P}}{1-v^{2}}(\hat{\Omega}-\hat{\omega})
\end{aligned}
$$

where $s$ is an auxiliary radial coordinate defined in CST. The entire iterative scheme used to solve the approximate equations is identical to the one in CST.

To calibrate the approximation, we first compute an exact sequence of constant rest mass polytropes of increasing angular momentum. Each member of the sequence is specified by two parameters: the ratio of polar to equatorial radius, and the central rest-mass density. We next compute the approximate sequence using the same values for these two parameters for each model. We then compare the metric coefficients of corresponding models using the relationships in (35). We also compare global quantities such as the total mass and angular momentum. As a further diagnostic, we calculate two relativistic virial quantities $[11,12]$ whose values should be identically one for an exact equilibrium solution. In the notation of CST, these quantities are

$$
\begin{aligned}
& \lambda_{2 d}=\frac{32 \pi \int\left[P+(\epsilon+P) \frac{v^{2}}{1-v^{2}}\right] e^{2 \alpha} r d r d \theta}{\int\left\{\left(\frac{\partial \gamma}{\partial r}+\frac{\partial \rho}{\partial r}\right)^{2}+\frac{1}{r^{2}}\left(\frac{\partial \gamma}{\partial \theta}+\frac{\partial \rho}{\partial \theta}\right)^{2}-3 e^{-2 \rho} \sin ^{2} \theta\left[r^{2}\left(\frac{\partial \omega}{\partial r}\right)^{2}+\left(\frac{\partial \omega}{\partial \theta}\right)^{2}\right]\right\} r d r d \theta}, \\
& \lambda_{3 d}=16 \pi \int\left[3 P+(\epsilon+P) \frac{v^{2}}{1-v^{2}}\right] e^{2 \alpha+(\gamma-\rho) / 2} r^{2} \sin \theta d r d \theta /\left\{\int[\partial(\gamma+\rho) \partial(\gamma+\rho)\right. \\
& -\partial \alpha \partial \gamma+\partial \alpha \partial \rho-\frac{1}{2 r}\left(1-e^{2 \alpha-\gamma+\rho}\right)\left(4 \frac{\partial \alpha}{\partial r}+\frac{4}{r \tan \theta} \frac{\partial \alpha}{\partial \theta}-\frac{\partial \gamma}{\partial r}-\frac{1}{r \tan \theta} \frac{\partial \gamma}{\partial \theta}+\frac{\partial \rho}{\partial r}+\frac{1}{r \tan \theta} \frac{\partial \rho}{\partial \theta}\right) \\
& \left.\left.-\frac{3}{2} e^{-2 \rho} r^{2} \sin ^{2} \theta \partial \omega \partial \omega\right] e^{(\gamma-\rho) / 2} r^{2} \sin \theta d r d \theta\right\},
\end{aligned}
$$

where, for example,

$$
\partial \alpha \partial \rho \equiv \frac{\partial \alpha}{\partial r} \frac{\partial \rho}{\partial r}+\frac{1}{r^{2}} \frac{\partial \alpha}{\partial \theta} \frac{\partial \rho}{\partial \theta}
$$

and $\epsilon=\rho_{0}+\rho_{i}$ is the total mass-energy density. Here $\lambda_{3 d}$ involves an integration with a three-dimensional volume element $r^{2} \sin \theta d r d \theta$ and is the relativistic generalization of the classical virial theorem

$$
2 E_{\mathrm{kin}}+3(\Gamma-1) E_{\mathrm{int}}+U_{\mathrm{grav}}=0 .
$$

The quantity $\lambda_{2 d}$ involves an integration with a twodimensional volume element $r d r d \theta$. The discrepancy from unity is a measure of numerical error for our solutions of the exact equations. It is a measure of the larger inherent error for our solutions of the approximate equations.

\section{AXISYMMETRIC ROTATING STAR: NUMERICAL RESULTS}

To calibrate the approximate scheme against the exact solution, we choose the most stringent case, in which the configuration is very relativistic and rapidly rotating. When it is rotating rapidly, there are large deviations from spherical symmetry, so that the approximation is no longer exact. For polytropes, the largest rotation is attained for nearly incompressible matter, i.e., for large $\Gamma=1+1 / n$ or small polytropic index $n$. We choose $n=0.5$.

In constructing an exact sequence of rotating equilibria as 


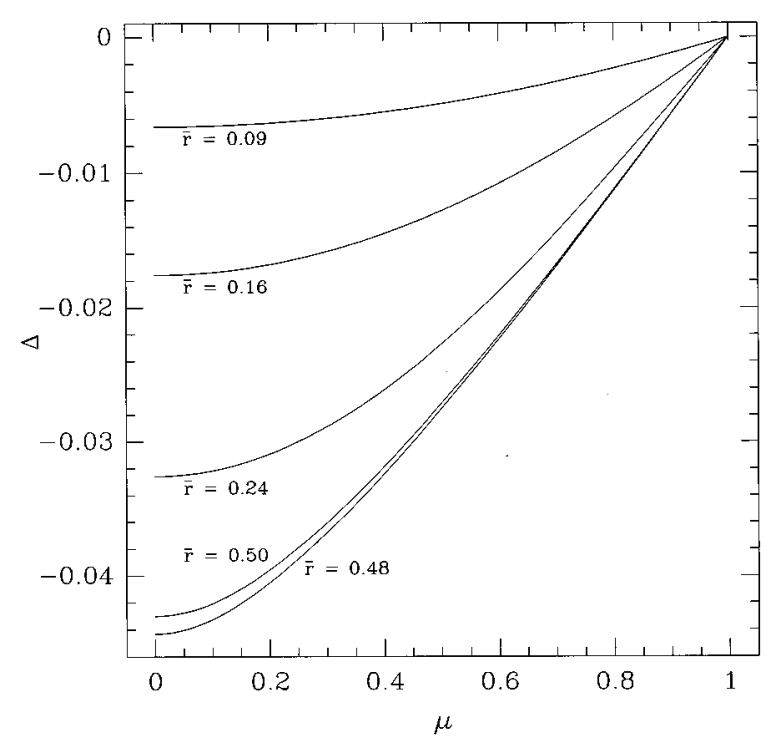

FIG. 1. Angular profile of the deviation of the exact solution from conformal flatness at selected radii. The deviation $\Delta$ is defined in Eq. (44). The star is a rapidly rotating, highly relativistic polytrope with $n=0.5$ and rest mass just below the maximum rest mass of a nonrotating star for this equation of state. The radii $\bar{r}$ are in the nondimensional units of CST, and $\mu=\cos \theta$.

a benchmark, we start with a nonrotating star having a central value of energy density $\bar{\epsilon}=1$ (note that all "barred" quantities are nondimensional as defined by CST). This configuration is relativistic, with $M / R=0.298$ and rest mass $\bar{M}_{0}=0.148$, just below the maximum rest mass of a nonrotating star for this equation of state $\left(\bar{M}_{0}=0.151\right)$. Holding the rest mass constant, we construct a sequence of increasing uniform rotation, up to the point of mass shedding. As described above, we then construct the corresponding models with the same central value of $\bar{\epsilon}$ and ratio of polar to equatorial radius using the approximate scheme. A comparison of some of the global quantities for the sequence is given in

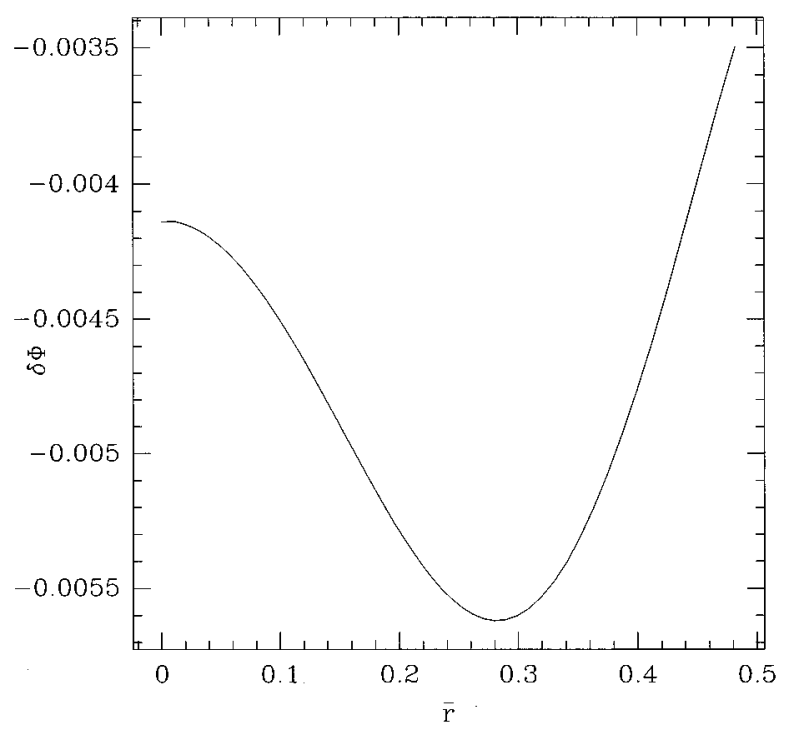

FIG. 2. Fractional error in the conformal factor $\Phi$ along an equatorial radius for the star in Fig. 1.

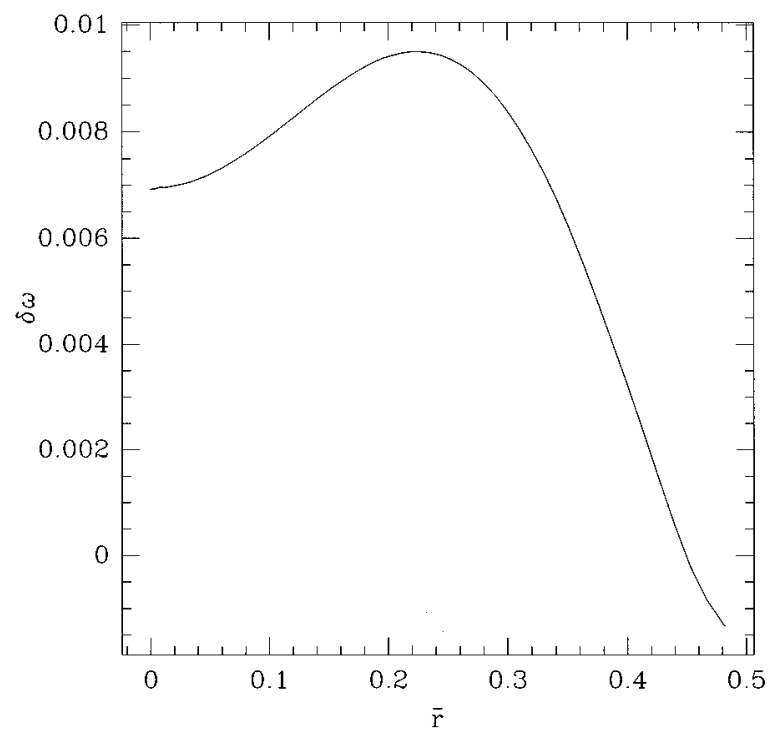

FIG. 3. Fractional error in the metric coefficient $\omega$ along an equatorial radius for the star in Fig. 1.

Table I. The high values of polar redshift $Z_{p}$ and $T / W$ confirm that the sequence is both highly relativistic and rapidly rotating. As expected, the deviations are largest near the mass shed limit, but even there they are never worse than about $1 \%$.

We can understand why the overall discrepancy is small by looking at Fig. 1. Here we plot a measure of the deviation in the exact solution from conformal flatness, which is assumed in the approximate method. In the figure we plot the angular profile at selected radii of the quantity

$$
\Delta \equiv \frac{\alpha_{\mathrm{CST}}-(\gamma-\rho) / 2}{\alpha_{\mathrm{CST}}}
$$

computed for the exact rotating model with $T / W=0.159$. Note that this quantity is identically zero on the axis because

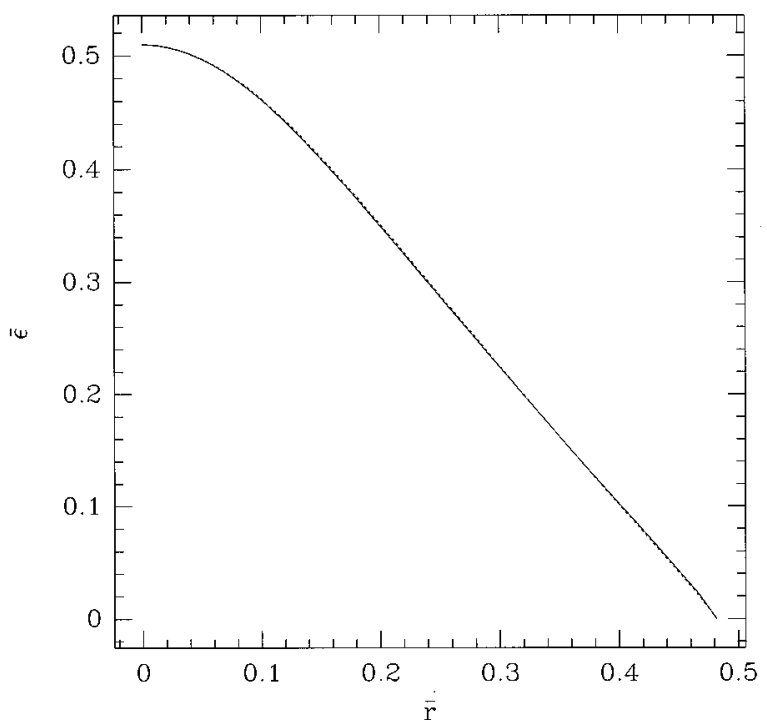

FIG. 4. Total mass-energy density $\bar{\epsilon}$ along an equatorial radius for the star in Fig. 1. The solid line shows the exact solution, the dotted line, the approximate solution. 
of local flatness there. The maximum deviation occurs on the equator $(\bar{r}=0.48)$, but is only about $5 \%$. Outside the star, $\Delta \rightarrow 0$ as $r \rightarrow \infty$.

In Fig. 2 we plot along an equatorial radius the fractional error in the conformal factor,

$$
\delta \Phi=\frac{\Phi-\Phi_{\text {exact }}}{\Phi_{\text {exact }}}
$$

where $\Phi_{\text {exact }} \equiv \exp [(\gamma-\rho) / 4]$. Similarly, in Fig. 3 we plot the fractional error $\delta \omega$. Figure 4 shows the mass-energy $\bar{\epsilon}$ along an equatorial radius for the two schemes. The two profiles are almost coincident.
A further comparison is provided by Fig. 5, which shows the virial quantities $\lambda_{2 d}$ and $\lambda_{3 d}$ along the sequence, computed for each of the two schemes. In the case of the exact method, the deviation from unity is a measure of numerical error, which is less than $0.1 \%$. The deviation for the approximate method measures the inherent error, which is about a factor of 10 bigger.

To push the approximate scheme to the limit, we now consider a second equilibrium sequence, a "supramassive", sequence. This sequence has no nonrotating member, since its rest mass exceeds the maximum rest mass of a nonrotating star for this equation of state $\left(\bar{M}_{0}=0.151\right)$. Thus the sequence exists only by virtue of rotation. We construct the supramassive sequence with $\bar{M}_{0}=0.176$. We expect the dis-

TABLE II. Quantities characterizing a "supramassive" evolutionary sequence of $n=0.5$ polytropic neutron star models. Entries are as described for Table I.

\begin{tabular}{|c|c|c|c|c|c|c|c|c|c|c|}
\hline $\bar{\epsilon}_{c}^{\mathrm{a}}$ & $\bar{\Omega}^{\mathrm{b}}$ & $\overline{I^{\mathrm{c}}}$ & $\bar{M}^{\mathrm{d}}$ & $\bar{M}_{0}^{\mathrm{e}}$ & $T / W^{\mathrm{f}}$ & $\bar{R}_{e}^{\mathrm{g}}$ & $e^{\mathrm{h}}$ & $\omega_{c} / \Omega_{c}{ }^{\mathrm{i}}$ & $v / c^{\mathrm{j}}$ & $Z_{p}{ }^{\mathrm{k}}$ \\
\hline 1.0957 & 0.9464 & 0.01552 & 0.1471 & 0.1758 & 0.1248 & 0.4819 & 0.703 & 0.8344 & 0.5196 & 0.9282 \\
\hline- & 0.9549 & 0.01525 & 0.1472 & 0.1760 & 0.1246 & 0.4760 & 0.714 & 0.8355 & 0.5192 & 0.9294 \\
\hline 1.0602 & 0.9362 & 0.01571 & 0.1472 & 0.1758 & 0.1254 & 0.4857 & 0.705 & 0.8284 & 0.5174 & 0.9132 \\
\hline- & 0.9444 & 0.01544 & 0.1472 & 0.1760 & 0.1251 & 0.4799 & 0.717 & 0.8295 & 0.5170 & 0.9144 \\
\hline 1.0258 & 0.9269 & 0.01592 & 0.1472 & 0.1758 & 0.1264 & 0.4901 & 0.709 & 0.8223 & 0.5163 & 0.8989 \\
\hline- & 0.9349 & 0.01566 & 0.1473 & 0.1760 & 0.1261 & 0.4843 & 0.720 & 0.8234 & 0.5158 & 0.9001 \\
\hline 0.9925 & 0.9182 & 0.01615 & 0.1473 & 0.1758 & 0.1277 & 0.4948 & 0.714 & 0.8162 & 0.5158 & 0.8851 \\
\hline - & 0.9260 & 0.01588 & 0.1473 & 0.1760 & 0.1274 & 0.4892 & 0.724 & 0.8172 & 0.5153 & 0.8861 \\
\hline 0.9603 & 0.9105 & 0.01640 & 0.1474 & 0.1758 & 0.1294 & 0.5003 & 0.720 & 0.8100 & 0.5166 & 0.8719 \\
\hline- & 0.9182 & 0.01614 & 0.1474 & 0.1760 & 0.1291 & 0.4946 & 0.730 & 0.8110 & 0.5162 & 0.8730 \\
\hline 0.9292 & 0.9031 & 0.01668 & 0.1475 & 0.1758 & 0.1314 & 0.5062 & 0.726 & 0.8037 & 0.5179 & 0.8591 \\
\hline- & 0.9107 & 0.01641 & 0.1476 & 0.1760 & 0.1311 & 0.5005 & 0.736 & 0.8048 & 0.5176 & 0.8602 \\
\hline 0.8991 & 0.8964 & 0.01698 & 0.1476 & 0.1758 & 0.1337 & 0.5127 & 0.734 & 0.7974 & 0.5203 & 0.8467 \\
\hline- & 0.9038 & 0.01671 & 0.1477 & 0.1760 & 0.1334 & 0.5071 & 0.743 & 0.7985 & 0.5200 & 0.8478 \\
\hline 0.8699 & 0.8901 & 0.01731 & 0.1478 & 0.1758 & 0.1365 & 0.5202 & 0.742 & 0.7911 & 0.5239 & 0.8348 \\
\hline- & 0.8975 & 0.01703 & 0.1479 & 0.1760 & 0.1362 & 0.5145 & 0.751 & 0.7922 & 0.5237 & 0.8361 \\
\hline 0.8417 & 0.8839 & 0.01766 & 0.1480 & 0.1758 & 0.1395 & 0.5284 & 0.752 & 0.7847 & 0.5283 & 0.8231 \\
\hline - & 0.8912 & 0.01738 & 0.1481 & 0.1760 & 0.1392 & 0.5228 & 0.760 & 0.7858 & 0.5282 & 0.8244 \\
\hline 0.8144 & 0.8779 & 0.01805 & 0.1482 & 0.1758 & 0.1429 & 0.5380 & 0.763 & 0.7783 & 0.5343 & 0.8117 \\
\hline- & 0.8852 & 0.01776 & 0.1483 & 0.1760 & 0.1426 & 0.5324 & 0.771 & 0.7794 & 0.5343 & 0.8129 \\
\hline 0.7880 & 0.8719 & 0.01847 & 0.1484 & 0.1758 & 0.1466 & 0.5493 & 0.775 & 0.7719 & 0.5423 & 0.8004 \\
\hline- & 0.8790 & 0.01817 & 0.1485 & 0.1759 & 0.1462 & 0.5438 & 0.782 & 0.7729 & 0.5424 & 0.8015 \\
\hline 0.7625 & 0.8654 & 0.01894 & 0.1487 & 0.1758 & 0.1506 & 0.5645 & 0.790 & 0.7654 & 0.5546 & 0.7891 \\
\hline- & 0.8723 & 0.01862 & 0.1487 & 0.1759 & 0.1500 & 0.5591 & 0.797 & 0.7663 & 0.5549 & 0.7898 \\
\hline 0.7593 & 0.8648 & 0.01901 & 0.1487 & 0.1758 & 0.1512 & 0.5677 & 0.793 & 0.7646 & 0.5579 & 0.7878 \\
\hline- & 0.8716 & 0.01868 & 0.1487 & 0.1759 & 0.1506 & 0.5623 & 0.800 & 0.7655 & 0.5583 & 0.7884 \\
\hline 0.7562 & 0.8637 & 0.01907 & 0.1487 & 0.1758 & 0.1517 & 0.5701 & 0.795 & 0.7637 & 0.5598 & 0.7863 \\
\hline - & 0.8705 & 0.01873 & 0.1487 & 0.1759 & 0.1510 & 0.5647 & 0.802 & 0.7646 & 0.5602 & 0.7867 \\
\hline 0.7531 & 0.8629 & 0.01914 & 0.1488 & 0.1758 & 0.1522 & 0.5740 & 0.799 & 0.7629 & 0.5639 & 0.7849 \\
\hline - & 0.8695 & 0.01878 & 0.1487 & 0.1758 & 0.1514 & 0.5687 & 0.805 & 0.7637 & 0.5644 & 0.7851 \\
\hline 0.7500 & 0.8620 & 0.01921 & 0.1488 & 0.1758 & 0.1528 & 0.5818 & 0.806 & 0.7621 & 0.5729 & 0.7835 \\
\hline- & 0.8682 & 0.01881 & 0.1487 & 0.1757 & 0.1517 & 0.5767 & 0.812 & 0.7627 & 0.5736 & 0.7828 \\
\hline
\end{tabular}

${ }^{a}$ Central energy density.

${ }^{\mathrm{b}}$ Angular velocity measured at infinity.

${ }^{\mathrm{c}}$ Moment of inertia.

${ }^{\mathrm{d}}$ Total mass energy.

${ }^{\mathrm{e}}$ Rest mass.

${ }^{\mathrm{f}}$ Rotational kinetic energy over gravitational binding energy.

${ }^{\mathrm{g}}$ Circumferential radius.

${ }^{\mathrm{h}}$ Eccentricity.

${ }^{\mathrm{i}}$ Measure of frame dragging.

${ }^{\mathrm{j}}$ Matter velocity at equator.

${ }^{\mathrm{k}}$ Polar redshift. 


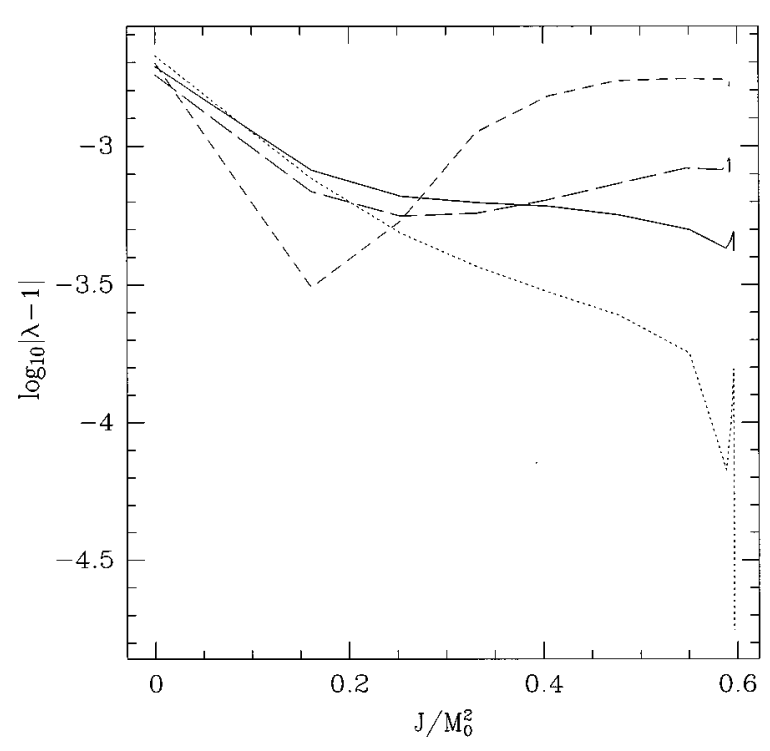

FIG. 5. Virial quantities along the sequence in Table I. Results for the exact equations are shown by the solid line for $\lambda_{2 d}$ and the dotted line for $\lambda_{3 d}$. Results for the approximation are shown by the short-dashed line for $\lambda_{2 d}$ and the long-dashed line for $\lambda_{3 d}$.

crepancy between the approximate and exact methods to be somewhat larger for this sequence since it is everywhere far from spherical symmetry. This expectation is borne out in Table II and Fig. 6. Nevertheless, the discrepancy is not very large.

\section{CONCLUSION}

We have tested Wilson's approximation scheme on rapidly rotating relativistic stars. Since these are equilibrium objects, it is necessary that the scheme give reasonably accurate results if we are to believe its predictions for more complicated systems such as binaries. In fact, we have found that the method works remarkably well, even for highly relativistic objects far from spherical symmetry. The largest er-

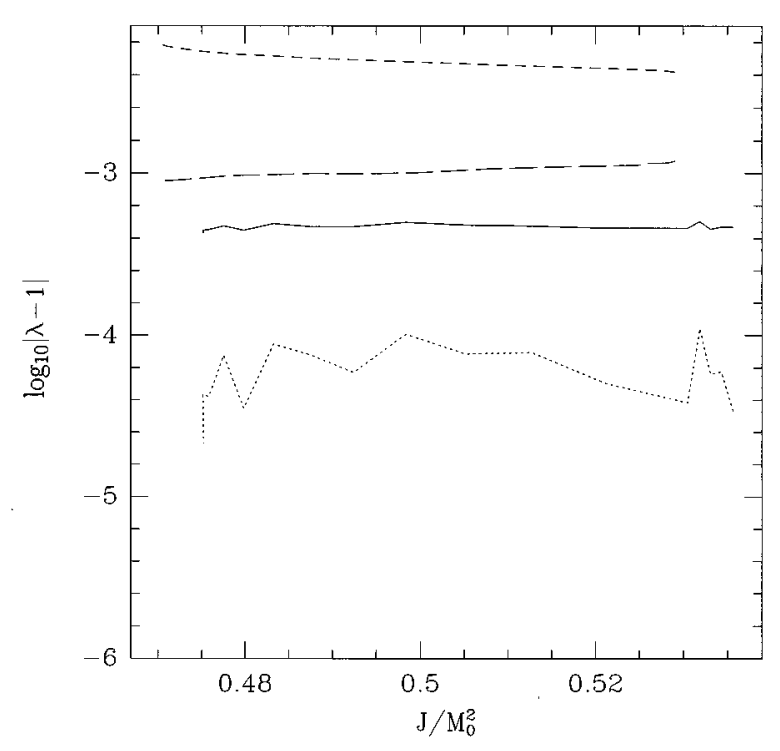

FIG. 6. Virial quantities along the supramassive sequence in Table II. Results for the exact equations are shown by the solid line for $\lambda_{2 d}$ and the dotted line for $\lambda_{3 d}$. Results for the approximation are shown by the short-dashed line for $\lambda_{2 d}$ and the long-dashed line for $\lambda_{3 d}$.

rors in any quantities we examined were around 5\%, and in general the errors were much smaller. Global measures such as virial quantities were in error by far less than $1 \%$. This agreement is very encouraging.

\section{ACKNOWLEDGMENTS}

We thank E. Gourgoulhon for useful discussions about the work on relativistic virial quantities in Refs. [11,12]. This work was supported in part by NSF Grant Nos. AST 9119475 and PHY 94-08378, and by NASA Grant No. NAGW-2364 to Cornell University. We also acknowledge support from the Grand Challenge Grant No. NSF PHY 9318152/ASC 93-18152.
[1] J. R. Wilson and G. J. Mathews, in Frontiers in Numerical Relativity, edited by C. R. Evans, L. S. Finn, and D. W. Hobill (Cambridge University Press, Cambridge, England, 1989), pp. 306-314.

[2] J. R. Wilson, in Texas Symposium on 3-Dimensional Numerical Relativity, edited by R. A. Matzner (University of Texas, Austin, Texas, 1990).

[3] J. R. Wilson and G. J. Mathews, Phys. Rev. Lett. 75, 4161 (1995).

[4] Other approximations for binary black holes have been presented by Detweiler [13] and Cook [14].

[5] Note that we are free to choose $\gamma^{-1 / 3} \gamma_{i j}$ to be any timeindependent metric. The choice of a flat metric simplifies the derivations slightly and results in familiar flat-space differential operators.

[6] A. P. Lightman, W. H. Press, R. H. Price, and S. A. Teukolsky, Problem Book in Relativity and Gravitation (Princeton Univer- sity Press, Princeton, New Jersey, 1975).

[7] G. B. Cook, S. L. Shapiro, and S. A. Teukolsky, Astrophys. J. 398, 203 (1992).

[8] G. B. Cook, S. L. Shapiro, and S. A. Teukolsky, Astrophys. J. 422, 227 (1994).

[9] G. B. Cook, S. L. Shapiro, and S. A. Teukolsky, Astrophys. J. 424, 823 (1994).

[10] Note that some minor errors in Ref. [7] were corrected in Ref. [8].

[11] E. Gourgoulhon and S. Bonazzola, Class. Quantum Gravit. 11, 443 (1994).

[12] S. Bonazzola and E. Gourgoulhon, Class. Quantum Gravit. 11, 1775 (1994).

[13] J. K. Blackburn and S. Detweiler, Phys. Rev. D 46, 2318 (1992).

[14] G. B. Cook, Phys. Rev. D 50, 5025 (1994). 